\title{
Association between the Type of Diabetes Treatment and Depressive Symptoms among Patients with Diabetes: A Cross-Sectional Study of Korea Community Health Surveys Data, 2011-2016
}

\author{
Hyeon Ji Lee ${ }^{1,2}{ }^{\oplus}$, Jieun Jang ${ }^{1,2}$, Sang Ah Lee ${ }^{3 \oplus}$, Sarah Soyeon $\mathrm{Oh}^{1,2}{ }^{1}$ and \\ Eun-Cheol Park $2,4, *$ (iD \\ 1 Department of Public Health, Graduate School, Yonsei University, Seoul 03722, Korea; \\ leehj612@yuhs.ac (H.J.L.); JIEUN99@yuhs.ac (J.J.); sarahoh@yuhs.ac (S.S.O.) \\ 2 Institute of Health Services Research, Yonsei University, Seoul 03722, Korea \\ 3 Research and Analysis Team, National Health Insurance Service Ilsan Hospital, Goyang 10444, Korea; \\ laurenleey91@gmail.com \\ 4 Department of Preventive Medicine, College of Medicine, Yonsei University, Seoul 03722, Korea \\ * Correspondence: ECPARK@yuhs.ac; Tel.: +82-2-2228-1862
}

Received: 27 October 2019; Accepted: 12 November 2019; Published: 12 November 2019

\begin{abstract}
The purpose of this study was to examine the association between the different types of treatment for diabetes and depressive symptoms. In particular, this study assessed the presence of depressive symptoms in patients with diabetes who are undergoing pharmacological treatments in terms of sex. This study used data from the 2011-2016 Korea Community Health Survey, which included responses from 50,774 male and 48,978 female participants with diabetes who were receiving pharmacological treatments. Patients aged $\geq 30$ years were included. Logistic regression analysis was conducted to examine the significance of the association. Male participants treated with insulin injection were more likely to experience depressive symptoms than those taking oral hypoglycemic (oral agents) only (odds ratio $(\mathrm{OR})=1.27 ; 95 \%$ confidence interval $(\mathrm{CI}): 1.04-1.56)$. Male patients treated with both oral agents and insulin injection had the highest OR value of depressive symptoms among different types of treatments (OR $=1.41,95 \% \mathrm{CI}: 1.25-1.60)$. The same tendency was observed in female participants. In female patients, however, the association between depressive symptoms and insulin injection was statistically insignificant (both oral agents and insulin injection $\mathrm{OR}=1.35$, $95 \%$ CI: $1.22-1.50$, insulin injection OR $=1.17,95 \%$ CI: 0.98-1.41). The association between depressive symptoms and the type of diabetes treatment was more significant in male than in female patients. Those who were treated with oral agents and insulin injection were more likely to have depressive symptoms than those receiving oral agents of treatment.
\end{abstract}

Keywords: diabetes mellitus; depression; therapeutics; drug therapy; insulin

\section{Introduction}

Diabetes is a non-communicable disease [1]. However, its prevalence is increasing worldwide [2]. According to International Diabetes Federation (IDF) data, the number of individuals with diabetes worldwide in 2017 was approximately 424.9 million, with a prevalence rate of $8.8 \%$. Moreover, in 2045, its prevalence is predicted to increase up to $9.9 \%$ among 6.37 billion adults worldwide [3]. The increase in its prevalence is a cause of concern because diabetes is one of the major causes of death. According to the World Health Organization (WHO) data, diabetes is the sixth leading cause of death 
in 2015, with 1.59 million recorded cases [4]. The leading cause of death among diabetes patients is diabetes complications.

Blood glucose levels that are poorly managed increase the risk of these complications [5]. In other words, uncontrolled diabetes can cause complications, which can threaten the lives of patients. Therefore, the blood glucose levels (pharmacological or non-pharmacological treatment) of patients with diabetes must be controlled. If the blood glucose levels of patients with diabetes is properly controlled, diabetes-related complications and death can be prevented [3].

In contrast, depression increases the risk of complications and death among individuals with diabetes. Depression is a well-known comorbidity in patients with chronic diseases, and diabetes is not an exception [6]. Distress from the condition, depressive mood or symptoms, and clinical depression can be observed in patients with diabetes [7]. Depressive symptoms in patients with diabetes reduces adherence to treatments [8] and disrupts the management of high blood glucose. Moreover, they can cause complications and increase the risk of mortality from diabetes [3,9]. Diabetes patients with depression have approximately 1.5 times increased risk of mortality compared to diabetes patients without depression [10]. Thus, depression is fatal in these patients.

In several previous studies, diabetes and depressive symptoms had a bidirectional association [11]. Both are risk factors that cause each other to occur, and they also exacerbate each other's disease state. Therefore, individuals with diabetes have twice the increased risk for depression than those without $[12,13]$. In particular, among patients with diabetes, emotional distress (including depressive symptoms) is more commonly observed in women than in men [14,15]. Approximately $80 \%$ of patients with diabetes are more likely to experience a recurrence of depressive symptoms [16]. Furthermore, treatments for high blood glucose had a negative impact on depression [17-20].

According to previous studies, the degree of depressive symptoms among patients with diabetes differed according to the type of treatment for diabetes. Studies that used the problem areas in diabetes (PAID) questionnaire focused on depression, and the emotional problems of the patients with diabetes showed different emotional distresses, which depends on the type of treatment for type 2 diabetes [15]. In another study that used the PAID questionnaire, the scores of the IDDM (insulin dependent diabetes mellitus) and NIDDM (non-insulin-dependent diabetes mellitus) groups differed in terms of the type of diabetes treatment [21]. In addition, in a study by Black on older Mexican Americans with diabetes, the current type of diabetes treatment was associated with depressive symptoms [22]. The study by Hermanns showed the difference in the proportion of individuals with type 1 and 2 diabetes without insulin treatment and those with type 2 diabetes with insulin treatment who were diagnosed with clinical and subclinical depression [23].

Therefore, the present study aimed to examine the association between the different types of diabetes treatment and depressive symptoms. In particular, the depressive symptoms of patients with diabetes who were on pharmacologic treatments (oral agents, insulin injection, and both oral agent medications and insulin injection) were assessed in terms of sex.

\section{Materials and Methods}

\subsection{Data Collection and Participants}

This study used data from The Korea Community Health Survey (CHS) in 2011-2016. The CHS is a cross-sectional, annual, nationwide public health survey, which has been performed by the Korea Center for Disease Control and Prevention (KCDC) since 2008. The surveyed population is registered adult residents aged 19 years and older in the community health center. The sample design is a complex, stratified, multistage, probability-cluster sampling design. The survey is conducted by a trained interviewer in a one-on-one interview in Korean through computer-assisted personal interview (CAPI) [24]. The CHS data are openly published. Additionally, participants' information was fully anonymized and unidentified prior to analysis. Accordingly, ethical approval was not required for this study. Ethical approval of the CHS was obtained from the KCDC (2010-02CON-22-P; 2011-05CON-04-C; 
2012-07CON-01-2C; 2013-06EXP-01-3C; 2014-08EXP-09-4C-A; 2016-10-01-P-A). The dataset is available on The CHS website (https://chs.cdc.go.kr/chs/index.do).

The total number of respondents in this survey was $1,372,650$. However, only respondents aged 30 years or older $(n=1,220,729)$ were selected. Two-stage filtering was performed to identify patients with diabetes who were undergoing treatment. First, patients who answered yes to the question "Have you ever been diagnosed with diabetes by a doctor?" were included $(n=128,474)$. Second, only those who answered yes to the question "Are you currently being treated?" were included among them $(n=115,990)$. There were no missing data in the questionnaire that asked for the types of diabetes treatment. Individuals with missing data on covariate variables were further excluded. Therefore, a final sample population of 99,752 was included in the analysis. Of these, 50,774 were male and 48,978 were female.

\subsection{Dependent Variable}

Self-reported depressive symptom was considered as a dependent variable. The following question was included in the questionnaire: "Have you ever felt sad or desperate enough to cause disruption to your daily life over two consecutive weeks in the last year?" The respondents were classified who answered yes as having depressive symptoms and those who answered no as having no depressive symptoms. Those who responded with "Don't know" and "No response" were excluded.

\subsection{Independent Variable}

The type of diabetes treatment used to control blood glucose was considered an independent variable. This study used the CHS question "What is the treatment you are doing to manage your blood glucose? Please answer all" to set the interest variable. The possible responses to the questions include the following: insulin injections (yes, no), oral agent medications (oral agents; yes, no), non-pharmacologic therapy (exercise, diet; yes, no). Among the patients, those who answered that they were only treated with non-pharmacological therapy were excluded to examine the association between the different type of pharmacological diabetes treatments and depression.

\subsection{Control Variables}

The covariates were as follows: age, socioeconomic (SES) factors, health-related factors, and the year of the survey. For SES factors, educational level, marital status, occupation (white collar, pink collar, blue collar, and others), monthly household income, and region of residence were considered. The monthly household income was categorized into six groups. At the start of the investigation, $1000 \mathrm{KRW}$ was equivalent to 0.93 USD [25]. Health-related factors included alcohol status (whether they drink more than one cup of alcohol during their lifetime), smoking status (whether they smoke more than five packs during their lifetime), walking activities, perceived health status, perceived stress, body mass index, and a history or diagnosis of hypertension and dyslipidemia. Walking activities were assessed by using the following questionnaire: "How many days did you walk at least 10 minutes at a time in the last week?" The responses were classified as follows: None or 1 day per week as inactive, 2-3 days a week as low, 4-5 days a week as middle, and 6-7 days a week as high.

\subsection{Statistical Analysis}

$\chi^{2}$ test and logistics regression analysis were used for the analysis. All the analyses were performed by classifying the patients according to sex (male and female). $\chi^{2}$ test was used to examine the significant difference in depressive symptoms depending on the different types of pharmacological treatments for diabetes and to control covariates, such as age, SES factors, health-related factors, and year. A $p$ value $<0.05$ was considered statistically significant. Logistic regression analysis was conducted to determine adjusted odds ratios (ORs) and 95\% confidence intervals (CIs). Subgroup analysis was performed according to the different types of treatments for diabetes and depressive 
symptoms. Statistical analyses were performed by using the Statistical Analysis System software version 9.4 (SAS Institute Inc., Cary, NC, USA).

\section{Results}

\subsection{Study Population}

Table 1 shows the general characteristics of the study population. Of the total diabetes patients who were undergoing diabetes treatment, 90,182 (90.4\%) were treated with oral agents and $7049(7.1 \%)$ were treated with oral agents and insulin injection. Insulin injection was the least used $(2521 ; 2.5 \%)$. Among the male and female diabetes patients, 2911 (2.9\%) and 5207 people (5.2\%) had depressive symptoms, respectively, indicating a higher proportion of women affected. As for the depressive symptoms according to the type of treatment, in men, treatment with both oral agents and insulin injection were the most frequently used at $5.7 \%$, followed by insulin injection at $5.1 \%$. Oral agents were the least frequently used at $2.6 \%$. In women, treatment with both oral agents and insulin injection were the most frequently used at $8.2 \%$, followed by insulin injection at $6.2 \%$. Oral agents were the least frequently used at $5.0 \%$.

Table 1. General characteristics of the study population with depressive symptoms.

\begin{tabular}{|c|c|c|c|c|c|c|c|c|c|c|c|c|}
\hline \multirow{3}{*}{ Variables } & \multirow{2}{*}{\multicolumn{2}{|c|}{ Total }} & \multicolumn{4}{|c|}{ Male } & \multirow{3}{*}{$p$-Value * } & \multicolumn{4}{|c|}{ Female } & \multirow{3}{*}{$p$-Value * } \\
\hline & & & \multicolumn{2}{|c|}{ Yes } & \multicolumn{2}{|c|}{ No } & & \multicolumn{2}{|c|}{ Yes } & \multicolumn{2}{|c|}{ No } & \\
\hline & $N$ & $\%$ & $N$ & $\%$ & $N$ & $\%$ & & $N$ & $\%$ & $N$ & $\%$ & \\
\hline Total & 99,752 & 100.0 & 2911 & 2.9 & 47,863 & 48.0 & & 5207 & 5.2 & 43,771 & 43.9 & \\
\hline Diabetes treatment & & & & & & & $<0.0001$ & & & & & $<0.0001$ \\
\hline Oral agents & 90,182 & 90.4 & 2384 & 2.6 & 43,415 & 48.1 & & 4471 & 5.0 & 39,912 & 44.3 & \\
\hline Insulin injection & 2521 & 2.5 & 128 & 5.1 & 1273 & 50.5 & & 157 & 6.2 & 963 & 38.2 & \\
\hline $\begin{array}{l}\text { Oral agents and insulin } \\
\text { injection }\end{array}$ & 7049 & 7.1 & 399 & 5.7 & 3175 & 45.0 & & 579 & 8.2 & 2896 & 41.1 & \\
\hline Age & & & & & & & $<0.0001$ & & & & & 0.0006 \\
\hline $30-39$ & 1396 & 1.4 & 79 & 5.7 & 780 & 55.9 & & 67 & 4.8 & 470 & 33.7 & \\
\hline $40-49$ & 7528 & 7.5 & 286 & 3.8 & 4402 & 58.5 & & 343 & 4.6 & 2497 & 33.2 & \\
\hline $50-59$ & 21,613 & 21.7 & 738 & 3.4 & 11,794 & 54.6 & & 1011 & 4.7 & 8070 & 37.3 & \\
\hline $60-69$ & 32,185 & 32.3 & 850 & 2.6 & 15,683 & 48.7 & & 1592 & 4.9 & 14,060 & 43.7 & \\
\hline $70-79$ & 29,785 & 29.9 & 793 & 2.7 & 12,594 & 42.3 & & 1774 & 6.0 & 14,624 & 49.1 & \\
\hline $80 \sim$ & 7245 & 7.3 & 165 & 2.3 & 2610 & 36.0 & & 420 & 5.8 & 4050 & 55.9 & \\
\hline Educational level & & & & & & & $<0.0001$ & & & & & 0.2868 \\
\hline Elementary school or less & 44,717 & 44.8 & 879 & 2.0 & 12,627 & 28.2 & & 3337 & 7.5 & 27,874 & 62.3 & \\
\hline Middle school & 18,166 & 18.2 & 607 & 3.3 & 9650 & 53.1 & & 822 & 4.5 & 7087 & 39.0 & \\
\hline High school & 24,168 & 24.2 & 925 & 3.8 & 15,642 & 64.7 & & 831 & 3.4 & 6770 & 28.0 & \\
\hline College or over & 12,701 & 12.7 & 500 & 3.9 & 9944 & 78.3 & & 217 & 1.7 & 2040 & 16.1 & \\
\hline Marital status & & & & & & & $<0.0001$ & & & & & $<0.0001$ \\
\hline Married-cohabit & 72,774 & 73.0 & 2022 & 2.8 & 41,690 & 57.3 & & 2768 & 3.8 & 26,294 & 36.1 & \\
\hline $\begin{array}{l}\text { Married non-cohabitant or } \\
\text { bereaved or divorced }\end{array}$ & 25,153 & 25.2 & 716 & 2.8 & 4972 & 19.8 & & 2371 & 9.4 & 17,094 & 68.0 & \\
\hline Unmarried & 1825 & 1.8 & 173 & 9.5 & 1201 & 65.8 & & 68 & 3.7 & 383 & 21.0 & \\
\hline Occupation & & & & & & & $<0.0001$ & & & & & $<0.0001$ \\
\hline White collar & 7244 & 7.3 & 246 & 3.4 & 5855 & 80.8 & & 90 & 1.2 & 1053 & 14.5 & \\
\hline Pink collar & 8424 & 8.4 & 164 & 1.9 & 3838 & 45.6 & & 392 & 4.7 & 4030 & 47.8 & \\
\hline Blue collar & 32,561 & 32.6 & 752 & 2.3 & 20,694 & 63.6 & & 826 & 2.5 & 10,289 & 31.6 & \\
\hline Others & 51,523 & 51.7 & 1749 & 3.4 & 17,476 & 33.9 & & 3899 & 7.6 & 28,399 & 55.1 & \\
\hline $\begin{array}{l}\text { Monthly household } \\
\text { income, } 1000 \mathrm{KRW}\end{array}$ & & & & & & & $<0.0001$ & & & & & $<0.0001$ \\
\hline$\sim 999$ & 35,161 & 35.2 & 1370 & 3.9 & 12,982 & 36.9 & & 2683 & 7.6 & 18,126 & 51.6 & \\
\hline 1000-1999 & 22,848 & 22.9 & 603 & 2.6 & 11,569 & 50.6 & & 1092 & 4.8 & 9584 & 41.9 & \\
\hline 2000-2999 & 15,729 & 15.8 & 377 & 2.4 & 8401 & 53.4 & & 627 & 4.0 & 6324 & 40.2 & \\
\hline 3000-3999 & 10,287 & 10.3 & 242 & 2.4 & 5714 & 55.5 & & 321 & 3.1 & 4010 & 39.0 & \\
\hline $4000-4999$ & 6181 & 6.2 & 120 & 1.9 & 3535 & 57.2 & & 212 & 3.4 & 2314 & 37.4 & \\
\hline $5000 \sim$ & 9546 & 9.6 & 199 & 2.1 & 5662 & 59.3 & & 272 & 2.8 & 3413 & 35.8 & \\
\hline Residency region & & & & & & & $<0.0001$ & & & & & $<0.0001$ \\
\hline Urban area-large city & 23,897 & 24.0 & 833 & 3.5 & 11,457 & 47.9 & & 1458 & 6.1 & 10,149 & 42.5 & \\
\hline Urban area-small city & 18,580 & 18.6 & 592 & 3.2 & 8790 & 47.3 & & 1041 & 5.6 & 8157 & 43.9 & \\
\hline Rural area & 57,275 & 57.4 & 1486 & 2.6 & 27,616 & 48.2 & & 2708 & 4.7 & 25,465 & 44.5 & \\
\hline Alcohol status & & & & & & & 0.5162 & & & & & $<0.0001$ \\
\hline Ever & 73,066 & 73.2 & 2640 & 3.6 & 43,232 & 59.2 & & 3074 & 4.2 & 24,120 & 33.0 & \\
\hline Never & 26,686 & 26.8 & 271 & 1.0 & 4631 & 17.4 & & 2133 & 8.0 & 19,651 & 73.6 & \\
\hline
\end{tabular}


Table 1. Cont.

\begin{tabular}{|c|c|c|c|c|c|c|c|c|c|c|c|c|}
\hline \multirow{3}{*}{ Variables } & \multirow{2}{*}{\multicolumn{2}{|c|}{ Total }} & \multicolumn{4}{|c|}{ Male } & \multirow{3}{*}{$p$-Value * } & \multicolumn{4}{|c|}{ Female } & \multirow{3}{*}{$p$-Value * } \\
\hline & & & \multicolumn{2}{|c|}{ Yes } & \multicolumn{2}{|c|}{ No } & & \multicolumn{2}{|c|}{ Yes } & \multicolumn{2}{|c|}{ No } & \\
\hline & $N$ & $\%$ & $N$ & $\%$ & $N$ & $\%$ & & $N$ & $\%$ & $N$ & $\%$ & \\
\hline Smoking status & & & & & & & 0.0476 & & & & & $<0.0001$ \\
\hline Ever & 44,517 & 44.6 & 2413 & 5.4 & 38,972 & 87.5 & & 623 & 1.4 & 2509 & 5.6 & \\
\hline Never & 55,235 & 55.4 & 498 & 0.9 & 8891 & 16.1 & & 4584 & 8.3 & 41,262 & 74.7 & \\
\hline Walking activity & & & & & & & $<0.0001$ & & & & & $<0.0001$ \\
\hline Inactive & 29,997 & 30.1 & 1062 & 3.5 & 14,062 & 46.9 & & 1851 & 6.2 & 13,022 & 43.4 & \\
\hline Low & 15,902 & 15.9 & 438 & 2.8 & 7364 & 46.3 & & 873 & 5.5 & 7227 & 45.4 & \\
\hline Middle & 13,996 & 14.0 & 372 & 2.7 & 6453 & 46.1 & & 711 & 5.1 & 6460 & 46.2 & \\
\hline High & 39,857 & 40.0 & 1039 & 2.6 & 19,984 & 50.1 & & 1772 & 4.4 & 17,062 & 42.8 & \\
\hline Perceived health status & & & & & & & $<0.0001$ & & & & & $<0.0001$ \\
\hline Good & 13,026 & 13.1 & 160 & 1.2 & 8543 & 65.6 & & 190 & 1.5 & 4133 & 31.7 & \\
\hline Normal & 34,556 & 34.6 & 628 & 1.8 & 19,126 & 55.3 & & 890 & 2.6 & 13,912 & 40.3 & \\
\hline Bad & 52,170 & 52.3 & 2123 & 4.1 & 20,194 & 38.7 & & 4127 & 7.9 & 25,726 & 49.3 & \\
\hline Perceived stress level & & & & & & & $<0.0001$ & & & & & $<0.0001$ \\
\hline Very & 25,887 & 26.0 & 1863 & 7.2 & 10,076 & 38.9 & & 3443 & 13.3 & 10,505 & 40.6 & \\
\hline Less & 45,860 & 46.0 & 832 & 1.8 & 22,838 & 49.8 & & 1412 & 3.1 & 20,778 & 45.3 & \\
\hline Low & 28,005 & 28.1 & 216 & 0.8 & 14,949 & 53.4 & & 352 & 1.3 & 12,488 & 44.6 & \\
\hline Body mass index $\left(\mathrm{kg} / \mathrm{m}^{2}\right)$ & & & & & & & $<0.0001$ & & & & & $<0.0001$ \\
\hline Underweight ( 18.4) & 2959 & 3.0 & 163 & 5.5 & 996 & 33.7 & & 295 & 10.0 & 1505 & 50.9 & \\
\hline Normal weight (18.5-22.9) & 33,982 & 34.1 & 1040 & 3.1 & 15,412 & 45.4 & & 1794 & 5.3 & 15,736 & 46.3 & \\
\hline Overweight (23.0-24.9) & 27,470 & 27.5 & 754 & 2.7 & 14,260 & 51.9 & & 1198 & 4.4 & 11,258 & 41.0 & \\
\hline Obesity $(25.0 \sim)$ & 35,341 & 35.4 & 954 & 2.7 & 17,195 & 48.7 & & 1920 & 5.4 & 15,272 & 43.2 & \\
\hline Hypertension diagnosis & & & & & & & 0.0027 & & & & & 0.0732 \\
\hline Yes & 60,434 & 60.6 & 1752 & 2.9 & 27,451 & 45.4 & & 3379 & 5.6 & 27,852 & 46.1 & \\
\hline No & 39,318 & 39.4 & 1159 & 2.9 & 20,412 & 51.9 & & 1828 & 4.6 & 15,919 & 40.5 & \\
\hline Dyslipidemia diagnosis & & & & & & & $<0.0001$ & & & & & $<0.0001$ \\
\hline Yes & 32,578 & 32.7 & 1078 & 3.3 & 13,732 & 42.2 & & 2308 & 7.1 & 15,460 & 47.5 & \\
\hline No & 67,174 & 67.3 & 1833 & 2.7 & 34,131 & 50.8 & & 2899 & 4.3 & 28,311 & 42.1 & \\
\hline Year & & & & & & & $<0.0001$ & & & & & $<0.0001$ \\
\hline 2011 & 14,080 & 14.1 & 362 & 2.6 & 6660 & 47.3 & & 627 & 4.5 & 6431 & 45.7 & \\
\hline 2012 & 14,856 & 14.9 & 351 & 2.4 & 7209 & 48.5 & & 663 & 4.5 & 6633 & 44.6 & \\
\hline 2013 & 16,061 & 16.1 & 462 & 2.9 & 7753 & 48.3 & & 812 & 5.1 & 7034 & 43.8 & \\
\hline 2014 & 17,230 & 17.3 & 591 & 3.4 & 8204 & 47.6 & & 1042 & 6.0 & 7393 & 42.9 & \\
\hline 2015 & 18,280 & 18.3 & 582 & 3.2 & 8746 & 47.8 & & 993 & 5.4 & 7959 & 43.5 & \\
\hline 2016 & 19,245 & 19.3 & 563 & 2.9 & 9291 & 48.3 & & 1070 & 5.6 & 8321 & 43.2 & \\
\hline
\end{tabular}

${ }^{*} p$-value is the result of the chi square test. Oral agents, Oral hypoglycemics; KRW, Korean Won;

\subsection{Association between Type of Diabetes Treatment and Depressive Symptoms}

Table 2 shows the results of the factors associated with the depressive symptoms of the diabetes patients. The logistic regression analyses adjusted for covariates revealed that the type of diabetes treatment was related to the depressive symptoms of the diabetes patients. In addition, the type of diabetes treatment was more closely related to the symptoms of depression in men with diabetes than in women with diabetes. Compared with taking oral agents, in both men and women, insulin injections more likely caused depressive symptoms. However, the results of women were not significant as compared with those of men (OR $=1.27 ; 95 \%$ CI: $1.04-1.56$ vs OR $=1.17,95 \%$ CI: $0.98-1.41)$. The male and female diabetes patients who received oral agents and insulin injections were the most likely to have depressive symptoms than the diabetes patients with one type of treatment. This showed statistically significant results for both men and women (OR $=1.41,95 \%$ CI: $1.25-1.60$ and $\mathrm{OR}=1.35$, $95 \%$ CI: $1.22-1.50$, respectively). 
Table 2. Factors associated with depressive symptoms in diabetes patients.

\begin{tabular}{|c|c|c|c|c|}
\hline \multirow{2}{*}{ Variables } & \multicolumn{2}{|c|}{ Male } & \multicolumn{2}{|c|}{ Female } \\
\hline & Adjusted OR & $95 \% \mathrm{CI}$ & Adjusted OR & $95 \% \mathrm{CI}$ \\
\hline \multicolumn{5}{|l|}{ Diabetes treatment } \\
\hline Oral agents & 1.00 & - & 1.00 & - \\
\hline Insulin injection & 1.27 & $(1.04-1.56)$ & 1.17 & $(0.98-1.41)$ \\
\hline Oral agents and insulin injection & 1.41 & $(1.25-1.60)$ & 1.35 & $(1.22-1.50)$ \\
\hline \multicolumn{5}{|l|}{ Age } \\
\hline 30-39 & 1.00 & - & 1.00 & - \\
\hline $40-49$ & 0.71 & $(0.54-0.95)$ & 1.20 & $(0.88-1.62)$ \\
\hline $50-59$ & 0.73 & $(0.56-0.97)$ & 1.17 & $(0.87-1.56)$ \\
\hline $60-69$ & 0.63 & $(0.48-0.84)$ & 0.92 & $(0.68-1.24)$ \\
\hline $70-79$ & 0.58 & $(0.44-0.78)$ & 0.82 & $(0.61-1.11)$ \\
\hline $80 \sim$ & 0.44 & $(0.32-0.62)$ & 0.70 & $(0.51-0.96)$ \\
\hline \multicolumn{5}{|l|}{ Educational level } \\
\hline Elementary school or less & 1.00 & - & 1.00 & - \\
\hline Middle school & 1.01 & $(0.90-1.14)$ & 1.05 & $(0.96-1.15)$ \\
\hline High school & 0.96 & $(0.86-1.08)$ & 1.12 & $(1.01-1.24)$ \\
\hline College or over & 0.90 & $(0.79-1.04)$ & 1.10 & $(0.92-1.31)$ \\
\hline \multicolumn{5}{|l|}{ Marital status } \\
\hline Married-cohabit & 1.00 & - & 1.00 & - \\
\hline Married non-cohabitant or bereaved or divorced & 1.93 & $(1.74-2.13)$ & 1.40 & $(1.31-1.50)$ \\
\hline Unmarried & 1.41 & $(1.15-1.72)$ & 1.26 & $(0.94-1.68)$ \\
\hline \multicolumn{5}{|l|}{ Occupation } \\
\hline White collar & 1.00 & - & 1.00 & - \\
\hline Pink collar & 0.92 & $(0.74-1.13)$ & 1.14 & $(0.88-1.48)$ \\
\hline Blue collar & 0.86 & $(0.72-1.02)$ & 1.12 & $(0.87-1.45)$ \\
\hline Others & 1.81 & $(1.52-2.16)$ & 1.78 & $(1.39-2.27)$ \\
\hline \multicolumn{5}{|l|}{ Monthly household income, $1000 \mathrm{KRW}$} \\
\hline$\sim 999$ & 1.00 & - & 1.00 & - \\
\hline 1000-1999 & 0.69 & $(0.62-0.77)$ & 0.82 & $(0.76-0.89)$ \\
\hline 2000-2999 & 0.63 & $(0.55-0.72)$ & 0.73 & $(0.66-0.81)$ \\
\hline 3000-3999 & 0.58 & $(0.49-0.68)$ & 0.58 & $(0.50-0.66)$ \\
\hline $4000-4999$ & 0.48 & $(0.38-0.59)$ & 0.71 & $(0.60-0.83)$ \\
\hline $5000 \sim$ & 0.49 & $(0.40-0.58)$ & 0.61 & $(0.53-0.71)$ \\
\hline \multicolumn{5}{|l|}{ Residency region } \\
\hline Urban area-large city & 1.00 & - & 1.00 & - \\
\hline Urban area-small city & 0.84 & $(0.75-0.95)$ & 0.84 & $(0.77-0.92)$ \\
\hline Rural area & 0.74 & $(0.67-0.81)$ & 0.74 & $(0.69-0.80)$ \\
\hline \multicolumn{5}{|l|}{ Alcohol status } \\
\hline Ever & 1.00 & - & 1.00 & - \\
\hline Never & 0.90 & $(0.78-1.03)$ & 0.89 & $(0.83-0.95)$ \\
\hline \multicolumn{5}{|l|}{ Smoking status } \\
\hline Ever & 1.00 & - & 1.00 & - \\
\hline Never & 1.10 & $(0.99-1.23)$ & 0.67 & $(0.60-0.74)$ \\
\hline \multicolumn{5}{|l|}{ Walking activity } \\
\hline Inactive & 1.00 & - & 1.00 & - \\
\hline Low & 0.94 & $(0.83-1.07)$ & 0.94 & $(0.86-1.03)$ \\
\hline Middle & 0.95 & $(0.83-1.09)$ & 0.95 & $(0.86-1.05)$ \\
\hline High & 0.86 & $(0.78-0.95)$ & 0.90 & $(0.83-0.97)$ \\
\hline \multicolumn{5}{|l|}{ Perceived health status } \\
\hline Good & 1.00 & - & 1.00 & - \\
\hline Normal & 1.44 & $(1.21-1.72)$ & 1.14 & $(0.97-1.35)$ \\
\hline Bad & 2.53 & $(2.13-3.00)$ & 1.90 & $(1.63-2.23)$ \\
\hline \multicolumn{5}{|l|}{ Perceived stress level } \\
\hline Very & 1.00 & - & 1.00 & - \\
\hline Less & 0.24 & $(0.22-0.26)$ & 0.24 & $(0.22-0.25)$ \\
\hline Low & 0.09 & $(0.08-0.11)$ & 0.10 & $(0.09-0.11)$ \\
\hline
\end{tabular}


Table 2. Cont.

\begin{tabular}{lcccc}
\hline \multirow{2}{*}{ Variables } & \multicolumn{2}{c}{ Male } & \multicolumn{2}{c}{ Female } \\
\cline { 2 - 5 } & Adjusted OR & 95\% CI & Adjusted OR & $\mathbf{9 5 \% ~ C I ~}$ \\
\hline Body mass index $\left(\mathbf{k g} / \mathbf{m}^{2}\right)$ & & & & \\
$\quad$ Underweight $(18.4)$ & 1.51 & $(1.24-1.84)$ & 1.45 & $(1.25-1.68)$ \\
$\quad$ Normal weight $(18.5-22.9)$ & 1.00 & - & 1.00 & - \\
Overweight (23.0-24.9) & 0.92 & $(0.83-1.02)$ & 0.96 & $(0.88-1.04)$ \\
Obesity (25.0 ) & 0.92 & $(0.83-1.02)$ & 0.98 & $(0.91-1.06)$ \\
\hline Hypertension diagnosis & & & & \\
Yes & 1.00 & - & 1.00 & - \\
No & 1.04 & $(0.96-1.14)$ & 1.06 & $(0.99-1.13)$ \\
\hline Dyslipidemia diagnosis & & & & \\
Yes & 1.00 & - & 1.00 & - \\
No & 0.75 & $(0.69-0.82)$ & 0.78 & $(0.73-0.84)$ \\
\hline Year & & & & \\
2011 & 1.00 & - & 1.00 & - \\
2012 & 0.89 & $(0.76-1.04)$ & 1.06 & $(0.94-1.19)$ \\
2013 & 1.15 & $(0.99-1.34)$ & 1.31 & $(1.16-1.47)$ \\
2014 & 1.36 & $(1.18-1.57)$ & 1.58 & $(1.42-1.77)$ \\
2015 & 1.31 & $(1.13-1.51)$ & 1.42 & $(1.27-1.59)$ \\
2016 & 1.17 & $(1.01-1.36)$ & 1.52 & $(1.36-1.70)$ \\
\hline
\end{tabular}

Oral agents, Oral hypoglycemics; KRW, Korean Won.

\subsection{Results of Subgroup Analyses}

Table 3 shows the results of the subgroup analyses of the association of age, educational level, walking activity, and body mass index with depressive symptoms. In most cases, in both men and women, insulin injection showed higher OR values than the reference value for oral agents, but many results of the subgroup analyses were not significant. In both men and women, oral agents and insulin injection together had higher OR values than the reference value for oral agents, with most of the patients showing significant results. The male patients treated with oral agents and insulin injection showed that the age of 40 to 49 had the highest $O R$ values $(O R=1.89,95 \% C I: 1.28-2.81)$ and the age of 60-69 had the lowest OR values (OR = 1.28, 95\% CI: 1.02-1.62). In body mass index, the OR value of underweight was not significant and that of normal weight was the lowest $(\mathrm{OR}=1.36,95 \% \mathrm{CI}$ : 1.11-1.67). The female treated with oral agents and insulin injection showed a similar trend to the male except for body mass index. The age of $40-49$ had the highest OR value $(\mathrm{OR}=1.54,95 \% \mathrm{CI}$ : 1.04-2.28), and the results for the age of 60-69 were not significant. In body mass index, the OR value of underweight was not significant. Normal weight had the highest OR value $(\mathrm{OR}=1.46,95 \% \mathrm{CI}$ : 1.22-1.74), overweight had a lower OR value (OR = 1.35, 95\% CI: 1.08-1.67), and obesity had the lowest $\mathrm{OR}$ value $(\mathrm{OR}=1.27,95 \% \mathrm{CI}$ : 1.07-1.50). This was a tendency different from the results of men.

Table 3. Subgroup analysis of depressive symptoms with the type of diabetes treatment by covariates *.

\begin{tabular}{|c|c|c|c|c|c|}
\hline \multirow{3}{*}{ Variables } & \multicolumn{5}{|c|}{ Diabetes Treatment } \\
\hline & \multirow{2}{*}{$\begin{array}{c}\text { Oral Agents } \\
\text { OR }\end{array}$} & \multicolumn{2}{|c|}{ Insulin Injection } & \multicolumn{2}{|c|}{ Oral Agents and Insulin Injection } \\
\hline & & OR & $95 \%$ CI & OR & $95 \% \mathrm{CI}$ \\
\hline \multicolumn{6}{|c|}{ Male } \\
\hline \multicolumn{6}{|l|}{ Age } \\
\hline 30-39 & + & + & & + & \\
\hline $40-49$ & 1.00 & 0.98 & $(0.51-1.89)$ & 1.89 & $(1.28-2.81)$ \\
\hline $50-59$ & 1.00 & 1.17 & $(0.78-1.74)$ & 1.53 & $(1.19-1.96)$ \\
\hline $60-69$ & 1.00 & 1.47 & $(1.02-2.10)$ & 1.28 & $(1.02-1.62)$ \\
\hline $70-79$ & 1.00 & 1.26 & $(0.84-1.91)$ & 1.34 & $(1.05-1.70)$ \\
\hline $80 \sim$ & - & & & & \\
\hline
\end{tabular}


Table 3. Cont.

\begin{tabular}{|c|c|c|c|c|c|}
\hline \multirow{3}{*}{ Variables } & \multicolumn{5}{|c|}{ Diabetes Treatment } \\
\hline & \multirow{2}{*}{$\begin{array}{c}\text { Oral Agents } \\
\text { OR }\end{array}$} & \multicolumn{2}{|c|}{ Insulin Injection } & \multicolumn{2}{|c|}{ Oral Agents and Insulin Injection } \\
\hline & & OR & $95 \% \mathrm{CI}$ & OR & $95 \% \mathrm{CI}$ \\
\hline \multicolumn{6}{|l|}{ Educational level } \\
\hline Elementary school or less & + & + & & + & \\
\hline Middle school & 1.00 & 1.38 & $(0.90-2.12)$ & 1.36 & $(1.03-1.78)$ \\
\hline High school & 1.00 & 1.53 & $(1.10-2.12)$ & 1.53 & $(1.23-1.91)$ \\
\hline College or over & 1.00 & 0.95 & $(0.55-1.65)$ & 1.75 & $(1.30-2.35)$ \\
\hline \multicolumn{6}{|l|}{ Walking activity } \\
\hline Inactive & 1.00 & 1.31 & $(0.94-1.84)$ & 1.55 & $(1.27-1.88)$ \\
\hline Low & 1.00 & 1.32 & $(0.76-2.32)$ & 1.46 & $(1.03-2.06)$ \\
\hline Middle & 1.00 & 1.07 & $(0.60-1.89)$ & 1.49 & $(1.04-2.13)$ \\
\hline High & 1.00 & 1.27 & $(0.91-1.78)$ & 1.22 & $(0.98-1.52)$ \\
\hline \multicolumn{6}{|l|}{ Body mass index $\left(\mathrm{kg} / \mathrm{m}^{2}\right)$} \\
\hline Underweight $(\sim 18.4)$ & 1.00 & 2.13 & $(1.00-4.54)$ & 1.66 & $(0.98-2.79)$ \\
\hline Normal weight (18.5-22.9) & 1.00 & 1.33 & $(0.98-1.81)$ & 1.36 & $(1.11-1.67)$ \\
\hline Overweight (23.0-24.9) & 1.00 & 1.33 & $(0.88-2.02)$ & 1.46 & $(1.13-1.89)$ \\
\hline Obesity $(25.0 \sim)$ & 1.00 & 0.96 & $(0.62-1.50)$ & 1.41 & $(1.13-1.76)$ \\
\hline \multicolumn{6}{|c|}{ Female } \\
\hline \multicolumn{6}{|l|}{ Age } \\
\hline $30-39$ & + & + & & + & \\
\hline $40-49$ & 1.00 & 1.22 & $(0.67-2.22)$ & 1.54 & $(1.04-2.28)$ \\
\hline $50-59$ & 1.00 & 1.03 & $(0.67-1.57)$ & 1.42 & $(1.12-1.80)$ \\
\hline $60-69$ & 1.00 & 1.30 & $(0.93-1.83)$ & 1.18 & $(0.98-1.42)$ \\
\hline $70-79$ & 1.00 & 1.21 & $(0.86-1.71)$ & 1.38 & $(1.15-1.64)$ \\
\hline $80 \sim$ & - & & & & \\
\hline \multicolumn{6}{|l|}{ Educational level } \\
\hline Elementary school or less & + & + & & + & \\
\hline Middle school & 1.00 & 1.26 & $(0.80-1.98)$ & 1.31 & $(1.01-1.70)$ \\
\hline High school & 1.00 & 1.09 & $(0.73-1.63)$ & 1.45 & $(1.12-1.88)$ \\
\hline College or over & 1.00 & 0.76 & $(0.36-1.60)$ & 1.84 & $(1.15-2.95)$ \\
\hline \multicolumn{6}{|l|}{ Walking activity } \\
\hline Inactive & 1.00 & 1.32 & $(0.98-1.78)$ & 1.45 & $(1.23-1.72)$ \\
\hline Low & 1.00 & 1.08 & $(0.66-1.76)$ & 1.39 & $(1.08-1.79)$ \\
\hline Middle & 1.00 & 1.80 & $(1.11-2.89)$ & 1.23 & $(0.91-1.66)$ \\
\hline High & 1.00 & 0.88 & $(0.62-1.24)$ & 1.24 & $(1.04-1.49)$ \\
\hline \multicolumn{6}{|l|}{ Body mass index $\left(\mathrm{kg} / \mathrm{m}^{2}\right)$} \\
\hline Underweight $(\sim 18.4)$ & 1.00 & 1.14 & $(0.56-2.35)$ & 1.27 & $(0.79-2.03)$ \\
\hline Normal weight (18.5-22.9) & 1.00 & 1.03 & $(0.76-1.40)$ & 1.46 & $(1.22-1.74)$ \\
\hline Overweight (23.0-24.9) & 1.00 & 1.28 & $(0.87-1.88)$ & 1.35 & $(1.08-1.67)$ \\
\hline Obesity $(25.0 \sim)$ & 1.00 & 1.30 & $(0.94-1.79)$ & 1.27 & $(1.07-1.50)$ \\
\hline
\end{tabular}

+: The number is insufficient and cannot be calculated; * Age, sex, educational level, marital status, occupation, monthly household income, residency region, alcohol status, smoking status, perceived health status, walking activity, stress level, body mass index, hypertension diagnosis, dyslipidemia diagnosis, and year variables were adjusted. Oral agents, Oral hypoglycemics;

\section{Discussion}

This study investigated the association between the types of treatment and depressive symptoms among diabetes patients undergoing diabetes treatment by dividing sex, using nationally represented data. The depressive symptoms in both male and female patients treated with diabetes varied according to the type of diabetes treatment. In all diabetes patients undergoing diabetes treatment, women reported more depressive symptoms than men. However, in examining the factors of depressive symptoms, it was found that men were more affected by depressive symptoms than women, depending on the type of diabetes treatment. As with the previous investigation, the overall likelihood of experiencing depressive symptoms in all the diabetes patients was higher for women 
than for men $[12,26]$. However, according to type of treatment, men had a greater risk of experiencing depressive symptoms than women [27].

In the male diabetes patients, in line with previous studies $[15,22,23]$, showed that treatment with "oral agent and insulin injection" or "insulin injection" was more likely to cause depressive symptoms than treatment with "oral agent" alone. Consistently with a previous study, the patients who were using insulin showed more depressive symptoms. In the Sample description section of the study by Hermanns, of 376 diabetes patients, 53 had clinical depression. Of these patients, 16 were receiving type 1 diabetes treatment (30.2\%); 11 were receiving type 2 diabetes treatment without insulin treatment $(20.8 \%)$; and 26 were receiving type 2 diabetes treatment with insulin $(49.0 \%)$. Of the 71 patients with subclinical depression, $31 \%, 22.5 \%$ and $46.5 \%$ were receiving the aforementioned treatments, respectively [23].

In female diabetes patients, oral agents and insulin injection significantly caused more depressive symptoms than oral agents alone according to statistics. Statistically significant results for insulin injection were difficult to obtain. However, the characteristics of the study population in our study showed that treatment with insulin injection resulted in a higher proportion of patients with depressive symptoms than treatment with oral agents. Previous studies showed that treatment with insulin injection affects depressive symptoms in diabetes patients. In a study that used PAID questionnaires to investigate the treatment methods for type 2 diabetes patients and their relationship to emotional distresses, the mean PAID score was 14.7 in the "diet alone" group and 17.8 in the "oral medication" group. "Insulin" had the highest score of 24.6 [15]. In Black's study of older Mexican American diabetes patients, "insulin" had the highest OR value (OR = 1.83; 95\% CI: 1.30-2.59), followed by "both insulin and OHA (oral hypoglycemic agent)" (OR = 1.79; 95\% CI: 1.10-2.89). "OHAs" had the lowest OR value (OR $=0.72 ; 95 \%$ CI: $0.51-0.99$ ) [22].

Our results for the male patients showed that the OR was highest for the oral agents and insulin injection group. That is, patients who took both oral agent medication and insulin injection tended to show depressive symptoms more often than patients who were treated only with insulin. In Welch's study that used PAID, the mean PAID score was lowest for "diet treated" at $10.5 \pm 12.8$ and highest for "IDDM" at $32.9 \pm 20.2$. For the "NIDDM" groups, including "insulin treated" $(26.4 \pm 20.8)$ and "tablet treated" $(24.9 \pm 22.1)$, the score was $25.0 \pm 20.9$ [21]. Hermanns reported that PAID questionnaires are useful to examine the depression and emotional problems of diabetes patients [23]. Therefore, depressive symptoms in patients taking oral medications should not be overlooked. In conclusion, in this study, the diabetes patients who were undergoing diabetes treatment only showed differences in the degree of depressive symptoms depending on their sex and type of diabetes treatment, and all were vulnerable to the risk of depressive symptoms.

This study performed subgroup analyses of depressive symptoms and type of diabetes treatments according to age and body mass index. The results showed that the diabetes patient had a stronger association with depressive symptoms if they used both oral agents and insulin injection. Both male and female patients who received oral agents and insulin injection treatment were most likely to experience depressive symptoms at the age of $40-49$ years. This is consistent with previous studies that showed that depression was more prevalent among diabetes patients aged 40-49 years [28]. Body mass index showed a different trend in the male and female patients. The normal-weight men had the lowest prevalence of depressive symptoms, while the women had the highest prevalence of depressive symptoms. Women were at higher and lower risks of experiencing depression when they were normal-weight and obese, respectively. This is considered to have been affected by the mental stress of the female to achieve normal weight due to the climate of Korean society, in which women care more about their weight than men.

Diabetes patients with depression have a lower quality of life and are less active in disease management than diabetes patients without depression [8,29]. Low quality of life and poor management of diseases worsens the depressive symptoms of diabetes patients with depression and eventually leads to a vicious circle that continues to degrade their health and quality of life. Therefore, managing 
depression in diabetics is very important. Our research is meaningful because we have again emphasized depression in diabetes patients by observing the association between the type of diabetes treatment and depressive symptoms among patients with diabetes. In addition, the bidirectional relationship between diabetes and depressive symptoms is well known [11]. However, regardless of whether depression adversely affects diabetes or diabetes adversely affects depression, all diabetes patients need treatments to manage their blood glucose. Therefore, our study is significant in that it demonstrates the association between the type of diabetes treatment and depressive symptoms. Furthermore, the strength of our study is that the depressive symptoms of diabetes patients were studied according to sex. Our research has the strength of its results being generalizable to the Korean adult population, as it used nationally representative data.

However, this study has some limitations, as it is conducted only with the aforementioned data. First, due to the bidirectional relationship between diabetes and depression [11], depression may occur before diabetes onset or treatment. On the contrary, diabetes can lead to or exacerbate depression, which can be caused by the burden of the disease (time, cost, effort) as well as by pathophysiological mechanisms $[15,30]$. Furthermore, if a patient with diabetes neglects to manage diabetes due to depression, the symptoms of diabetes may worsen. However, because the data is cross-sectional in design, the causal relationship between diabetes treatment and depressive symptoms remains unknown. In addition, due to the limitation of the survey items, it was not possible to know when diabetes and depression onset or when they were diagnosed and to know time, cost and efforts to manage diabetes. Therefore, it was not possible to detect patients who already had depression and to estimate the burden of disease in diabetes patients. Second, the presented data such as the depressive symptoms and health status variables were self-reported. Therefore, recall bias was possible. In addition, we used one question that asked about depressive symptoms rather than an objective depression inventory. Moreover, that question was about perceived depressive symptoms. Because there were no questions asked about the depression diagnosis or depression inventory, the responses to the feelings of sadness or depression for $>2$ weeks in a year were considered depressive symptoms. Third, the questions about diabetes were inadequate. None of the questions were about the type, duration, severity of diabetes, and glycemic control. Information about diabetes is important because it is closely related to the depressive symptoms and the strategy of treatment may vary accordingly. According to the Treatment Guideline for Diabetes in Korea, insulin injections are prescribed for type 1 diabetes, while oral hypoglycemic agents are prescribed first for type 2 diabetes, and if glycemic control is not good, use of both oral hypoglycemic agents and insulin injections is considered [31]. Therefore, type 2 diabetic patients who use both oral hypoglycemic agents and insulin injections may have more severe symptoms of diabetes. However, it was impossible to pinpoint the type and severity of diabetes in the data used in the study. In addition, diabetes duration is significantly associated with depression [32]. However, it could not be adjusted because it was also unknown. Moreover, our measures of the type of treatment for diabetes were based on single questions in the survey, which was too wide to know the details. Thus, the more detailed forms of insulin treatment (injection, pump [33]) or the ingredients and type of oral agents (metformin, thiazolidinediones, dipeptidyl peptidase-4, etc. [34]) could not be known. Also, how many times the patient took the oral agent or insulin injection could not be known. Finally, treatment efficacy according to disease management and medication adherence may be associated with depressive symptoms [35]. However, our study used cross-sectional data, and the questionnaire did not include the efficacy of the medicine, the severity of the disease, and the progress of the disease. Therefore, it is not possible to determine whether treatment efficacy influences the results. These limitations require further study.

The bidirectional association of depressive symptoms with diabetes treatment effects and adherence is also well recognized $[11,20]$. To prevent diabetes complications, blood glucose control should be performed well, and to maintain blood glucose at a proper level, the risk of depression should be avoided. Accordingly, physicians must also manage diabetes patients' mental health, including depressive symptoms. In addition, they should consider the depressive symptoms that diabetes 
patients may experience according to the type of diabetes treatment before the treatments are taken into account.

\section{Conclusions}

This study shows the difference in depressive symptoms according to the type of treatment among diabetes patients between the sexes. Depressive symptoms were more strongly associated with diabetes treatment in male patients than in female patients. Insulin injection was associated with depressive symptoms in diabetes patients. Oral agents and insulin injection had a significant correlation with depressive symptoms in diabetes patients. Oral agents and insulin injection were more likely to cause depressive symptoms than one form of treatment alone. Therefore, physicians should consider diabetes patients' risk for developing depressive symptoms according to sex and the type of diabetes treatment. They should also include a process that provides mental support and treatment when managing all diabetes patients.

Author Contributions: H.J.L. participated in conceptualization of the study, development of methodology, and writing the original draft. J.J. and S.A.L. participated in creation of models and analyzing the data. S.S.O. reviewed the manuscript. E.-C.P. is a guarantor of this study, providing oversight and leadership responsibility for the study. All authors read and approved the final manuscript.

Funding: This research did not receive any specific grant from funding agencies in the public, commercial, or not-for-profit sectors.

Acknowledgments: We are grateful to the Korea Center for Disease Control and Prevention for making use of the primary source, Korea Community Health Survey data.

Conflicts of Interest: The authors declare that there is no conflict of interest.

\section{References}

1. World Health Organization. Global Action Plan for the Prevention and Control of NCDs 2013-2020; World Health Organization: Geneva, Switzerland, 2013.

2. Kwon, H.S.; Kim, W.H.; Lee, D.Y.; Park, S.I. Current Status and Need for a Korean Diabetes Prevention Study; Public Health Weekly Report; Korea Centers for Disease Control and Prevention: Cheongju, Korea, 2015.

3. Karuranga, S.; Fernandes, J.R.; Huang, Y.; Malanda, B. Diabetes Atlas Eighth; International Diabetes Federation: Brussels, Belgium, 2017.

4. World Health Organization. The Top 10 Causes of Death; World Health Organization: Geneva, Switzerland, 2018; Available online: http://www.who.int/mediacentre/factsheets/fs310/en/ (accessed on 12 November 2019).

5. Fowler, M.J. Microvascular and macrovascular complications of diabetes. Clin. Diabetes. 2011, 26, 116-122. [CrossRef]

6. Myers, A.K.; Trivedi, M.H. Death by Insulin: Management of Self-Harm and Suicide in Diabetes Management. Curr. Diabetes Rev. 2017, 13, 251-262. [CrossRef] [PubMed]

7. Das Munshi, J.; Stewart, R.; Ismail, K.; Bebbington, P.E.; Jenkins, R.; Prince, M.J. Diabetes, common mental disorders, and disability: findings from the UK National Psychiatric Morbidity Survey. Psychosom. Med. 2007, 69, 543-550. [CrossRef] [PubMed]

8. Gonzalez, J.S.; Peyrot, M.; McCarl, L.A.; Collins, E.M.; Serpa, L.; Mimiaga, M.J.; Safren, S.A. Depression and diabetes treatment nonadherence: A meta-analysis. Diabetes Care 2008, 31, 2398-2403. [CrossRef] [PubMed]

9. Reichel, A.; Schwarz, J.; Schulze, J.; Licinio, J.; Wong, M.; Bornstein, S. Depression and anxiety symptoms in diabetic patients on continuous subcutaneous insulin infusion (CSII). Mol. Psychiatry 2005, 10, 975. [CrossRef] [PubMed]

10. Park, M.; Katon, W.J.; Wolf, F.M. Depression and risk of mortality in individuals with diabetes: A meta-analysis and systematic review. Gen. Hosp. Psychiatry 2013, 35, 217-225. [CrossRef] [PubMed]

11. Renn, B.N.; Feliciano, L.; Segal, D.L. The bidirectional relationship of depression and diabetes: A systematic review. Clin. Psychol. Rev. 2011, 31, 1239-1246. [CrossRef] [PubMed]

12. Nichols, G.A.; Brown, J.B. Unadjusted and adjusted prevalence of diagnosed depression in type 2 diabetes. Diabetes Care 2003, 26, 744-749. [CrossRef] [PubMed] 
13. Anderson, R.J.; Freedland, K.E.; Clouse, R.E.; Lustman, P.J. The prevalence of comorbid depression in adults with diabetes: A meta-analysis. Diabetes Care 2001, 24, 1069-1078. [CrossRef] [PubMed]

14. Jeon, H.S.; Cho, Y.M.; Hong, M.G. Depression, anxiety and its association on diabetes mellitus patients. J. Korean Acad. Fam. Med. 1996, 17, 83-90.

15. Delahanty, L.M.; Grant, R.W.; Wittenberg, E.; Bosch, J.L.; Wexler, D.J.; Cagliero, E.; Meigs, J.B. Association of diabetes-related emotional distress with diabetes treatment in primary care patients with type 2 diabetes. Diabet. Med. 2007, 24, 48-54. [CrossRef] [PubMed]

16. Katon, W.J. The comorbidity of diabetes mellitus and depression. Am. J. Med. 2008, 121, S8-S15. [CrossRef] [PubMed]

17. Rubin, R.R.; Peyrot, M. Psychological issues and treatments for people with diabetes. J. Clin. Psychol. 2001, 57, 457-478. [CrossRef] [PubMed]

18. Odegard, P.S.; Capoccia, K. Medication taking and diabetes. Diabetes Educ. 2007, 33, 1014-1029. [CrossRef] [PubMed]

19. Noh, J.H.; Park, J.K.; Lee, H.J.; Kwon, S.K.; Lee, S.H.; Park, J.H.; Ko, K.S.; Rhee, B.D.; Lim, K.H.; Kim, D.J. Depressive symptoms of type 2 diabetics treated with insulin compared to diabetics taking oral anti-diabetic drugs: A Korean study. Diabetes Res. Clin. Pract. 2005, 69, 243-248. [CrossRef] [PubMed]

20. Golden, S.H.; Lazo, M.; Carnethon, M.; Bertoni, A.G.; Schreiner, P.J.; Diez Roux, A.V.; Lee, H.B.; Lyketsos, C. Examining a bidirectional association between depressive symptoms and diabetes. JAMA 2008, 299, 2751-2759. [CrossRef] [PubMed]

21. Welch, G.W.; Jacobson, A.M.; Polonsky, W.H. The Problem Areas in Diabetes Scale: An evaluation of its clinical utility. Diabetes Care 1997, 20, 760-766. [CrossRef] [PubMed]

22. Black, S.A. Increased health burden associated with comorbid depression in older diabetic Mexican Americans. Results from the Hispanic Established Population for the Epidemiologic Study of the Elderly survey. Diabetes Care 1999, 22, 56-64. [CrossRef] [PubMed]

23. Hermanns, N.; Kulzer, B.; Krichbaum, M.; Kubiak, T.; Haak, T. How to screen for depression and emotional problems in patients with diabetes: comparison of screening characteristics of depression questionnaires, measurement of diabetes-specific emotional problems and standard clinical assessment. Diabetologia 2006, 49, 469-477. [CrossRef] [PubMed]

24. Kang, Y.W.; Ko, Y.S.; Kim, Y.J.; Sung, K.M.; Kim, H.J.; Choi, H.Y.; Sung, C.; Jeong, E. Korea community health survey data profiles. Osong Public Health Res. Perspect. 2015, 6, 211-217. [CrossRef] [PubMed]

25. Lee, Y.H. Socioeconomic differences among community-dwelling diabetic adults screened for diabetic retinopathy and nephropathy: The 2015 Korean Community Health Survey. PLoS ONE 2018, 13, e0191496. [CrossRef] [PubMed]

26. Lustman, P.J.; Griffith, L.S.; Clouse, R.E.; Cryer, P.E. Psychiatric illness in diabetes mellitus: Relationship to symptoms and glucose control. J. Nerv. Ment. Dis. 1986, 174, 736-742. [CrossRef] [PubMed]

27. Ali, S.; Stone, M.; Peters, J.; Davies, M.; Khunti, K. The prevalence of co-morbid depression in adults with Type 2 diabetes: a systematic review and meta-analysis. Diabet. Med. 2006, 23, 1165-1173. [CrossRef] [PubMed]

28. Brown, L.C.; Majumdar, S.R.; Newman, S.C.; Johnson, J.A. History of depression increases risk of type 2 diabetes in younger adults. Diabetes Care 2005, 28, 1063-1067. [CrossRef] [PubMed]

29. Goldney, R.D.; Phillips, P.J.; Fisher, L.J.; Wilson, D.H. Diabetes, depression, and quality of life: A population study. Diabetes Care 2004, 27, 1066-1070. [CrossRef] [PubMed]

30. Musselman, D.L.; Betan, E.; Larsen, H.; Phillips, L.S. Relationship of depression to diabetes types 1 and 2: epidemiology, biology, and treatment. Biol. Psychiatry 2003, 54, 317-329. [CrossRef]

31. Korean Diabetes Association. Treatment Guideline for Diabetes; Korean Diabetes Association: Seoul, Korea, 2019; pp. 2-169.

32. Rubin, R.R.; Peyrot, M. Quality of life and diabetes. Diabetes Metab. Res. Rev. 1999, 15, 205-218. [CrossRef]

33. Nuboer, R.; Borsboom, G.J.; Zoethout, J.A.; Koot, H.M.; Bruining, J. Effects of insulin pump vs. injection treatment on quality of life and impact of disease in children with type 1 diabetes mellitus in a randomized, prospective comparison. Pediatr. Diabete 2008, 9, 291-296. [CrossRef] [PubMed] 
34. Ko, S.H.; Kim, D.J.; Park, J.H.; Park, C.Y.; Jung, C.H.; Kwon, H.S.; Park, J.Y.; Song, K.H.; Han, K.; Lee, K.U.; et al. Trends of antidiabetic drug use in adult type 2 diabetes in Korea in 2002-2013: Nationwide population-based cohort study. Medicine (Baltimore) 2016, 95, e4018. [CrossRef] [PubMed]

35. Lin, E.H.; Katon, W.; Von Korff, M.; Rutter, C.; Simon, G.E.; Oliver, M.; Ciechanowski, P.; Ludman, E.J.; Bush, T.; Young, B. Relationship of depression and diabetes self-care, medication adherence, and preventive care. Diabetes Care 2004, 27, 2154-2160. [CrossRef] [PubMed]

(C) 2019 by the authors. Licensee MDPI, Basel, Switzerland. This article is an open access article distributed under the terms and conditions of the Creative Commons Attribution (CC BY) license (http://creativecommons.org/licenses/by/4.0/). 